\title{
EL CONO SUR LATINOAMERICANO Y LA INICIATIVA PARA LAS AMERICAS*
}

\author{
Francisco Rojas Aravena
}

En este trabajo se parte de la base de que en el sistema internacional se han producido profundas transformaciones y que en los procesos ya no están involucrados sólo asuntos de seguridad. Al mismo tiempo, los grandes cambios en América Latina -apertura económica y políticaobligan a buscar nuevas formas de cooperación, especialmente con la potencia hemisférica. Según el autor, por todo lo anterior, debe generarse un patrón de relación asociativo para enfrentar los desafíos, conciliando intereses y definiendo espacios de cooperación. En este contexto, y como una respuesta a la creación de bloques comerciales, se ubica la Iniciativa para las Américas. Explica en qué consiste ésta, lo que representa para Estados Unidos y las dificultades que ha tenido desde su formulación. Finalmente, se refiere a la reacción latinoamericana destacando el caso de Chile y los países del MERCosur, específicamente Argentina y Brasil. Concluye que factores en latinoamérica y Estados Unidos han restado peso político a la Iniciativa, dejándola subordinada al NAFTA.

Los cambios ocurridos en diversas partes del mundo en los últimos años han significado una profunda transformación en el sistema internacional. Esta no se ha reducido a una determinada área o tema, sino que abarca el conjunto de las relaciones y procesos. El término de la Guerra Fría se asocia en general al fin del conflicto bipolar y de las tensiones Este-Oeste. Sin embargo, en los procesos de post Guerra Fría se encuentran involucrados cambios que superan los asuntos tradicionales de la seguridad internacional. Los temas económicos y tecnológicos, las comunicaciones, las formas de producción y la necesidad de repensar el resurgimiento de viejos conflictos y aspiraciones nacionales, es lo característico de la nueva etapa.

Instituciones y organismos de la Guerra Fría han quedado vacíos de contenido. Las nuevas relaciones globales deben generar

\footnotetext{
*Trabajo presentado en el Segundo Congreso de Ciencia Política, Iquique, 24-27 noviembre de 1992 y en el VII PROFMEX-ANUIE International Conference "The Challenges of North-American Economic Integration", 11-14 noviembre de 1992, Mérida, México.
} 
sistemas de regulación adecuados a las transformaciones en curso y constituir regímenes internacionales que superen experiencias y conflictos del pasado reciente. Los fenómenos de la interdependencia global cada vez más consolidada requieren de mayor peso de las regulaciones internacionales. Parte importante de las dificultades de la hora presente está radicada en que los cambios no están totalmente decantados. Se perciben con claridad algunas tendencias, como el unipolarismo militar y la creciente marginación económica y estratégica de parte importante de los países de menor desarrollo ubicados en el Sur. Otras tendencias son más confusas, como las referidas a la constitución de bloques económicos cerrados en un mundo globalizado en aspectos centrales, como por ejemplo las comunicaciones y la producción.

El fin de la Guerra Fría ha permitido la emergencia de un importante número de conflictos de origen nacional, religioso, étni$\mathrm{co}$, territorial, que generan percepciones de inestabilidad, tensión y desorden.

Las incertidumbres y la inestabilidad en el plano global y regional se perciben como un rasgo de este período. Si bien los peligros de una conflagración mundial organizada en torno a dos grandes bloques ha desaparecido, la multiplicidad de pequeños conflictos genera percepciones de incertidumbre. Los propios procesos de globalización e interdependencia generan inestabilidades, al fraccionar internacionalmente tanto los procesos de producción como los mercados de consumo. La concentración de los circuitos financieros y la transferencia de recursos hacia los países más desarrollados dificulta la reactivación en el Sur. Los ciclos y ritmos asociados a productos y consumo de materias primas están cambiando con más velocidad, lo que afecta a los países del Sur que tienen procesos de adaptación tecnológica más lentos y es más débil su inserción global. El fin del conflicto Este-Oeste ha permitido ver la magnitud de las diferencias que separan al Norte desarrollado de los países del Sur.

En el mundo de post Guerra Fría, los márgenes de autonomía están fuertemente condicionados por el tipo de inserción y el posicionamiento económico internacional de cada Estado. Lo determinante en el próximo período, es la ubicación en las relaciones internacionales de mercado más que en las relaciones internacionales de seguridad. 
Este conjunto de cambios se expresa de manera profunda en el hemisferio occidental, donde también hay tensiones y procesos contradictorios. Estados Unidos es el único poder en la región y no hay poderes extraregionales que interfieran en su área de influencia. Sin embargo, no posee los recursos económicos para ayudar al desarrollo, lo que incide en la estabilidad económica y política en esa misma área. Los procesos de cambio en el Cono Sur latinoamericano se perciben rápidamente y, más aún, uno de los aportes que la región latinoamericana realizó al proceso de distensión mundial fue su proceso de redemocratización. América Latina fue capaz de desmilitarizar la política y reconstruir sistemas que se fundamentan en principios democráticos. Estamos en presencia de un consistente esfuerzo de apertura democrática, en el cual los ciudadanos eligen gobiernos - representativos en procesos que cobran cada día más transparencia. Las autoridades terminan sus mandatos y entregan el poder a quien resultó electo. Se ha avanzado hacia la estabilidad económica, meta que se ha alcanzado no sin dificultades en la mayoría de los países. Sin embargo, la reactivación es aún muy débil y los peligros de retroceso aparecen siempre como muy cercanos.

Si nos preguntamos qué es lo clave de los cambios en América Latina y el Cono Sur; debemos destacar dos cuestiones: i) La profundidad de la apertura económica y de los logros que ya se han obtenido en el proceso de inserción internacional. ii) La apertura política que permite la expresión de la democracia y un progresivo respeto a los derechos humanos básicos. Ambos procesos han sido concomitantes en el tiempo y han afectado la conceptualización, el tamaño y la forma de actuar del Estado en Latinoamérica. El ajuste estructural en la economía y la reforma política, han significado un proceso de cambio estatal de significación.

Desde mediados de la década pasada en la mayoría de los países de la región -en Chile con antelación- estaban en marcha planes y políticas de ajuste estructural. Abarcabando diversos aspectos, los centrales eran apertura económica, mayor incidencia de las regulaciones de mercado y una menor presencia estatal. Los procesos de ajuste se realizaron con una fuerte presencia y condicionamiento de los organismos multilaterales de crédito (Banco Mundial o Fondo Monetario Internacional) o de agencias de cooperación de gobiernos, en el caso de los países más pequeños (la AID para los Estados del Caribe y Centroamérica). Estos procesos debieron compatibilizar 
de manera contradictoria apertura e incentivos para la exportación, en medio de situaciones de desequilibrio macroeconómico y con altas tasas de inflación. ${ }^{1}$

América Latina debe conciliar valores nacionales y reforzar su soberanía, en un contexto en el cual la inserción internacional exige inevitablemente liberalización, apertura y formas de asociación y cooperación. Esto no sólo entre los países de la región sino en especial con su poder hegemónico.

La búsqueda de una agenda común que consolide esta doble apertura no ha dado, hasta la fecha, los frutos esperados. La concertación regional como pivote y refuerzo para los procesos nacionales ha sido débil y con una fuerte dosis de retórica. Los espacios y estructuras pensados para el acuerdo han servido fundamentalmente para el intercambio de información, compartir visiones o establecer diferencias. Este es un importante paso inicial, pero el peso de la concertación está en la definición conjunta de cursos de acción y en su puesta en marcha.

La forma y tipo de asociación, las tendencias integracionistas y los acuerdos de todo tipo, incidirán sobre las dos variables centrales -los procesos de apertura económica y política- que son las que determinan las tendencias claves para el futuro.

\section{Un patrón de relación asociativo.}

El fin de la Guerra Fría ha tenido un impacto que afecta a los conceptos bâsicos que organizaban los modelos teóricos, los parámetros de evaluación y el diseño de modelos para el deșarrollo de acciones. Las pautas que regulaban las acciones dejaron de tener vigencia, lo que hace necesario -en este contexto de confusión temporal-generar conceptos y modelos de carácter global que reemplacen los de la época de la Guerra Fria. El modelo básico surgido luego de la Segunda Guerra Mundial y que se mantuvo por cuatro décadas, fue el del conflicto permanente, global y multidimensional. Con el fin del conflicto ideológico es posible reorientar las relaciones

\footnotetext{
${ }^{1}$ Manuel R. Agosin, "Las experiencias de liberalización comercial en América Latina: Lecciones y Perspectivas", Pensamiento Iberoamericano, № 21, Madrid, España, 1992.

Roberto Bouzas, "Democracia, mercados y reformas estructurales en América Latina. El contexto económico internacional", FLACSO-Argentina, Ponencia Seminario, 1992.
} 
en el hemisferio, creando espacios para la asociación de políticas y el fomento de acciones de beneficio mutuo. Se trata de generar un patrón de relación asociativo.

Los procesos de redemocratización en Sudamérica, el triunfo del proceso de paz centroamericano de Esquipulas, los cambios de orientación que estableció desde sus inicios la administración Bush y el fin de la Guerra Fría, hicieron variar las prioridades hemisféricas. Por una parte, cambiaron las percepciones de amenaza y con ello se produjo un desplazamiento de la primacía de la esfera de la seguridad a otra donde la preeminencia la tienen las vinculaciones económicas. Por otra parte, las autoridades norteamericanas reconocieron que América Latina era interlocutor y actor, con la cual se debía concertar para buscar soluciones a los temas emergentes de la agenda global: medio ambiente, drogas, migraciones, comercio.

Surgió así la posibilidad de asociación, como elemento político crucial para enfrentar estos desafíos desde una nueva perspectiva, tanto en lo global y lo hemisférico como en lo regional. "El mundo está cambiando", señaló en 1990 el Subsecretario de Estado, Lawrence Eagleburger. Y añadió, "la eliminación de las tensiones EsteOeste nos permitirán enfocar con mayor claridad los problemas del Continente". Pero también y principalmente, porque "hemos terminado el debate rencoroso sobre América Central que nos distanció de nuestros vecinos y nos dividió en nuestra patria", señaló ante el Congreso, Bernard Aronson. ${ }^{2}$

Generar iniciativas que aborden los problemas centrales de los países del continente en los más diversos aspectos, es una necesidad de ayer y hoy. La diferencia está radicada en la posibilidad de pasar de un patrón de relación que tenía un fuerte peso en el unilateralismo, a otro de carácter asociativo. Los cambios globales y regionales establecen un espacio para la generación de alternativas que tienen la posibilidad de transformarse en el eje articulador de un nuevo patrón estratégico de relación entre Estados Unidos y los países del hemisferio, en especial los latinoamericanos. La asociación como modelo cobra cada vez mayor peso y factibilidad. En efecto, la insistencia y la búsqueda de un renovado diálogo entre Estados Unidos y los países de la región propuesto e impulsado por América

\footnotetext{
${ }^{2}$ Bernard Aronson, "Testimonio ante Comitć de Relaciones Exteriores del Senado de Estados Unidos", Usis, Embajada de Estados Unidos en Chile, 18 de abril de 1991.
} 
Latina, ha tenido una mejor receptividad en Washington. Luego de casi una década de distanciamiento, el Presidente Bush abrió una oportunidad al diálogo continental que posibilita establecer una común percepción de la realidad.

Una visión compartida, una definición de los intereses en juego y de los espacios de cooperación, es lo que posibilita la conformación de un nuevo patrón de relación basado en la asociación y construcción de intereses, a partir de la comprensión de las mutuas percepciones y su incorporación en las acciones diplomáticas. La conformación de este diagnóstico compartido se ha ido construyendo por medio de Cumbres Presidenciales de gran significación, como han sido la reunión hemisférica celebrada en Costa Rica (octubre de 1989), el encuentro con los presidentes del área andina en Cartagena (febrero de 1990) y luego en la Cumbre de San Antonio, y la gira latinoamericana del Presidente Bush (diciembre de 1990), además de las visitas de los mandatarios latinoamericanos a Washington. La cobertura hemisférica -una política de Alaska a la Antártida-cambia las orientaciones tradicionales en lo bilateral y en lo multilateral. La incorporación de Canadá a la Organización de Estados Americanos y el incremento de su presencia en la región, refuerzan la necesidad de hacer política hemisférica.

La Iniciativa para las Américas debe ser ubicada en este contexto. El diseño propuesto permite tener un marco de acción global, pero en el cual los diversos casos nacionales se insertan con sus propias particularidades y grandes diferencias. El marco temporal se adecua a esos lineamientos. Que el núcleo de la Iniciativa esté centrado en los aspectos de interrelación y de asociación -por medio del comercio, la inversión y la deuda-marcan un cambio de tendencia desde los factores tradicionales de poder hacia la interdependencia, en un sistema globalizado.

También, la Iniciativa hay que ubicarla en la formulación de una política preventiva frente a la creciente articulación de grandes macromercados que pudiesen dar pasos hacia la conformación de bloques. En este sentido, es parte de una red global de tratados entre los cuales los referidos a protección de inversiones, propiedad intelectual y servicios, conforman un cuerpo importante. ${ }^{3}$.

${ }^{3}$ The Joumal of Commerce, 24 agosto 1992. 


\section{La Iniciativa para las Américas.}

El 27 de junio de 1990, los embajadores latinoamericanos acreditados ante el gobierno norteamericano fueron citados con premura a la Casa Blanca. El Presidente Bush realizaría un anuncio de gran trascéndencia sobre las relaciones hemisféricas. En la oportunidad, anunció la Iniciativa para las Américas: una asociación para el comercio, las inversiones y el crecimiento. ${ }^{4}$

El Presidente analizó la situación internacional y hemisférica, indicando que hemos sido testigos de los grandes cambios mundiales y del resurgimiento de los gobiernos democráticos en el hemisferio. Las transformaciones en América Latina y el Caribe se desarrollan en forma paralela en la esfera económica y política, y el desafío para los Estados Unidos es responder apoyando los cambios positivos que están ocurriendo. "Debemos forjar una auténtica asociación para la reforma del libre mercado", señaló el Presidente. Ubicó el origen de esta Iniciativa en la Cumbre anti-drogas de Cartagena: "Salí de esa reunión convencido de que Estados Unidos debía revisar su enfoque no sólo hacia la región, sino también hacia América Latina y el Caribe como un todo" ... "la prosperidad en nuestro hemisferio depende del comercio, y no de la ayuda".

Seguidamente definió las bases de la Iniciativa. "Los tres pilares de la nueva Iniciativa son el comercio, las inversiones y la deuda. Para ampliar el comercio, propongo que comencemos el proceso de crear una zona de libre comercio a lo largo del hemisferio; para aumentar las inversiones, que adoptemos medidas para crear un nuevo flujo de capital hacia la región; y para aliviar más la carga de la deuda, un nuevo enfoque hacia la deuda en la región, con beneficios importantes para nuestro medio ambiente" ... "La gran lección económica de este siglo es que el proteccionismo paraliza el progreso, y que el libre mercado genera prosperidad". ${ }^{6}$

La Iniciativa se expresa en lo comercial en el objetivo de construir una zona de libre comercio que se extienda desde el Artico hasta el Polo Sur. Para ello, el primer paso es el acuerdo con México y la

\footnotetext{
${ }^{4}$ George Bush, "Discurso sobre las Nuevas Relaciones con el Hemisferio", Washington D.C., 27 de junio de 1990, Embajada de los Estados Unidos en Chile, junio de 1990.

${ }^{5}$ Ibid.

${ }^{6}$ Ibid.
} 
coordinación de políticas en la ronda Uruguay del GATT. En lo referente a las inversiones se busca generar un mecanismo multilateral que constaría de dos vertientes: la primera, establecería un fondo especial en el Banco Interamericano de Desarrollo (BID) para crear programas que faciliten la inversión externa. La segunda, es la creación de un Fondo Multilateral de Inversiones, al cual deberían concurrir Estados Unidos, Europa y Japón. Con respecto a la deuda, referida sólo a la oficial, se establece un mecanismo de canje de deuda por medidas sobre el medio ambiente.

En su discurso, el Presidente norteamericano destacó que los acontecimientos en Europa del Este no significarán que Estados Unidos pierdan de vista los tremendos desafíos y oportunidades que se generan en el hemisferio.

El camino elegido es incrementar la interdependencia económica, para lo cual se propone un programa que tiende a establecer una gran zona de libre comercio. Para ello es fundamental avanzar en los tres aspectos que conforman los pilares de la Iniciativa para las Américas: el comercio, la inversión y la deuda externa. ${ }^{7}$ Este camino significa avanzar hacia una "agenda positiva", es decir la búsqueda de una relación de asociación. Esto implica grandes avances para superar la "agenda negativa" o agenda de contención: evitar la influencia de poderes externos fundamentalmente por medios militares, más que desarrollar la interdependencia y la asociación.

La agenda positiva de los años noventa permite vincular los intereses de los distintos actores hemisféricos en torno al eje económico, particularmente el comercio y la inversión. La premisa es que la prosperidad depende del comercio y no de la ayuda. Ello significa que los actores privados -los empresarios, los inversionistas, los industriales, los exportadores, los importadores y los banqueros-son los que articulan las nuevas relaciones de mercado. Esto marca una importante diferencia con la Alianza para el Progreso, en la cual el eje era la ayuda y la cooperación que tenía como trasfondo los temas de seguridad, estableciendo un programa de contrainsurgencia. Es decir, los actores claves eran los gubernamentales y quienes se les oponían; eran éstos los que articulaban las relaciones internacionales de seguridad.

\footnotetext{
${ }^{7}$ Roger B. Porteer, "The Enterprise for the Americas Initiative a New Approach to Economic Growth", Joumal of Interamerican Studies and World Affairs, Vol. 32, No 4, Winter 1990.
} 
La Iniciativa para las Américas representa un cambio en la política norteamericana, ya que es una nueva perspectiva que tiende a poner énfasis en la relaciones de cooperación económica más que en los patrones tradicionales, en los que la seguridad tenían la preeminencia. Este cambio en las tendencias que orientan la política norteamericana hacia América Latina, reafirma su proceso de democratización y vuelve a poner al desarrollo y el crecimiento como una prioridad central.

Sin embargo, la Iniciativa también puede ser visualizada en la perspectiva de los intereses unilaterales estadounidenses. Ella conformaría parte de una visión más global y presupone, además, ciertas condiciones previas como estabilidad económica y ajuste estructural. Se establece una apertura simétrica, pero que en la práctica. será asimétrica y se desarrollará en forma bilateral en un contexto regional. El principal interés norteamericano es un reaseguro frente al fracaso del GATT, a la vez que asegura un área de influencia económica y un mercado liberalizado. A estos grandes objetivos se pueden agregar otros: asegurar las inversiones y revertir el déficit y la declinación de su comercio en un momento recesivo.

Sin embargo, a dos años de formulada, hay que señalar con claridad que la Iniciativa no logró transformarse en un eje de articulación de las políticas de Washington hacia América Latina. No se articuló el consenso suficiente para distribuir responsabilidades burocráticas, asignar fondos y establecer programas de acción. "Fuera del centro de Washington la Iniciativa para las Américas es aún un misterio". La rápida y en general positiva respuesta de América Latina no ha tenido una acogida similar en los círculos políticos y empresariales norteamericanos. "En el círculo íntimo de la Casa Blanca no hay una persona responsable directamente de la Iniciativa para las Américas, el Congreso es desatento, las corporaciones multinacionales han tenido un largo y público silencio, y los medios de comunicación le ha dedicado poca atención y espacio". ${ }^{8}$ La Iniciativa no logró tener una presencia política e intelectual de envergadura, como sí la tuvieron otras como la Alianza para el Progreso.

El impetu y la importancia con que surgió la Iniciativa fue declinando rápidamente. Las acciones de la presidencia norteameri-

\footnotetext{
${ }^{8}$ North-South Center, "Entrerprise for the Americas: Vision and Reality", North-South ISSUES, Universidad de Miami, julio de 1992.
} 
cana perdieron fuerza en el primer año. Muchas pueden ser las razones que expliquen esta situación, entre ellas claramente se pueden destacar: la prolongada y profunda recesión norteamericana, la campaña presidencial, el encontrarse en un ciclo en el que la importancia de la región en la agenda ha entrado en una "fase de olvido", la falta de éxito en acciones concretas de concertación hemisféricas como el caso de Haití. Todo ello ha redundado en que la Iniciativa para las Américas quedó como un enunciado sin contenido burocrático ni programa de acción. Esto ocurría a la par de una disminución drástica de la ayuda a la región, en especial a Centroamérica. El tratamiento del NAFTA pareció agotar los esfuerzos de la Casa Blanca a lo largo de 1992.

La falta de concreción de la política norteamericana quedó evidenciada de manera dramática en lo que se refiere a la creación del Fondo Multilateral de Inversiones, en donde pese a los reparos, finalmente, tanto los japoneses como algunos países europeos habían acordado realizar un aporte financiero similar al solicitado. Por su lado, Estados Unidos no aportó en forma oportuna su parte para la constitución del Fondo. Más aún, éste es muy pequeño y tendrá poco impacto, ya que es menor de lo que normalmente ingresa a los países de la región.?

Lo anterior nos muestra las dificultades que tiene Estados Unidos de concretar políticas globales hacia la región en la nueva etapa, en la cual el elemento organizador ya no es la seguridad. La fragmentación de la política norteamericana y la incidencia de múltiples actores parece conformar un dato de mayor reelevancia para el futuro. La primacía del comercio, la deuda y la inversión, hacen aparecer una variedad de intereses en juego que no son fáciles de concertar. En las décadas anteriores los temas económicos, en especial los tres mencionados, estaban subordinados a las definiciones estratégicas del ámbito de la seguridad. En la actual etapa, las nuevas redes de relación y los cambios en los pesos burocráticos de quienes toman las decisiones en Washington no se han decantado. La fragmentación en la política norteamericana tiene como consecuencia

\footnotetext{
${ }^{9}$ Evaluaciones periódicas de la Iniciativa para las Américas han sido realizadas por instituciones académicas y por consultoras privadas. En Chile, el Area de Relaciones Internacionales de FLACSO-Chile ha dado seguimiento a la Iniciativa. En Estados Unidos, pueden verse los trabajos dirigidos por Joseph Tulchin, Programa Latinoamericano de The Wilson Center, o los informes confidenciales sobre el tema de consultoras como Heller, Rosenblaatt \& Scheman.
} 
reafirmar cierta percepción de confusión e inmovilismo. La respuesta es tratar de generar nuevamente políticas generales y rearticular tendencias históricas de éxito, lo que hace recuperar fuerza al unilateralismo en un contexto internacional y sobre una agenda que requiere soluciones multilaterales basadas en una relación asociativa.

\section{La respuesta latinoamericana a la Iniciativa para las Américas.}

En América Latina por su parte, a medida que se avanzó en los estudios sobre el posible impacto de la Iniciativa para las Américas, las respuestas tendieron a diferenciarse. Inicialmente éstas se caracterizaron por: i) adhesión prematura, ii) incomprensión de los tiempos que requiere su implementación, iii) triunfalismo estridente. ${ }^{10}$ Sin embargo, al cumplirse el primer año de la Iniciativa ya era posible distinguir claramente respuestas diferenciadas en las diversas subregiones. El Caribe fue la región que miró en forma más cautelosa y mostró claramente falta de interés. Centroamérica fue cambiando crecientemente su entusiasmo inicial por la búsqueda de alternativas propias, centradas en su propia integración y en acuerdos con los poderes regionales que se expresan en esa zona. En Sudamérica las expectativas iniciales de rápidos avances y concreción de oportunidades se fueron perdiendo.

No se trata de una Iniciativa de aplicación homogénea para todos los países o áreas de la región, su aplicación está circunscrita a aquéllos que acepten los supuestos de una economía abierta y que establezcan en su legislación interna los pasos exigidos en materias de inversión, propiedad intelectual y otros por el gobierno norteamericano. ${ }^{11}$ Desde esta perspectiva se refuerza la bilateralidad que facilita la práctica de políticas unilaterales.

Las diversas respuestas latinoamericanas demuestran que no hay una una coincidencia natural de intereses, sino que éstos deben ser trabajados y organizados. La pregunta es dónde y cómo encontrar la compatibilidad, cuando no es evidente la tendencia a la coopera-

\footnotetext{
${ }^{10}$ Luis Maira, "Un examen de la Iniciativa para las Américas en el contexto del proceso político norteamericano", Documento de Trabajo, ILET, Santiago, 1992.

${ }^{11}$ Ibid. p. 36.
} 
ción y cuando los incentivos desde el mayor polo de poder apuntan hacia un privilegio de la bilateralidad. Los países de la región o de cada subregión son productores de similares materias primas o productos agrícolas que compiten en los mismos mercados internacionales. Lo anterior hace que la alternativa de la competencia y del "camino individual" surja con un mayor peso que el fomento a la cooperación. Por esta ruta lo que se pretende establecer es alguna característica que lo haga sujeto de una relación privilegiada. Es así como algunos se piensan como "modelos", otros se perciben como "el más confiable" y otros se ven como "estratégicos". Ello produce distorsiones que lejos de fomentar la colaboración distorsionan las metas, la articulación de intereses y la factibilidad de alcanzar objetivos satisfactorios para el conjunto.

Hoy, los factores centrales en América Latina son su propia percepción de apertura y cambio estructural y su renovada estabilidad como base del crecimiento. Su mayor debilidad es la falta de compatibilización de intereses y el establecimiento de una estrategia para alcanzarla, en un contexto de democracias débiles. Dada la apertura de América Latina su éxito está ligado al aumento del consumo internacional, al crecimiento de la demanda en los mercados de destino, a un mejoramiento y estabilidad en los precios, y a la apertura global. De allí que es muy significativo lo que ocurra en el GATr. Los acuerdos de libre comercio si bien son importantes no reemplazan a los logros que se deben alcanzar en el plano multilateral.

La respuesta diferencial que han dado los países del Cono Sur a la Iniciativa para las Américas evidencia lo señalado. A continuación se destacan las respuestas de Chile y los países del Mercosur. Se analiza en primer término la respuesta chilena, dado que fue señalado por las autoridades norteamericanas como el único país que en el actual fast track tenía posibilidades de ser incluido. Luego se exponen los casos de Argentina y Brasil.

\section{Chile y Estados Unidos: ¿Hacia un Acuerdo de Libre Comercio?}

Las relaciones con los Estados Unidos han concentrado y ocupado un lugar destacado de la atención del gobierno, de la elite política y 
del núcleo más dinámico del empresariado nacional chileno. Dada la importancia de los vínculos, su complejidad y la variedad de los temas en torno a los cuales se estructura la agenda, es posible mirar los intereses que expresan los diversos actores sociales y agentes económicos y analizar de qué forma se va conformando la política nacional en relación a la potencia hemisférica.

En los últimos años, se ha forjado en Chile un sólido consenso en torno a una estrategia de desarrollo basada en la apertura externa. La lucha contra el proteccionismo es uno de los objetivos permanentes de la política exterior. El desarrollo nacional exige consolidar los actuales mercados de exportación, otorgándoles estabilidad y seguridad, a la vez que se busca la expansión en otros nuevos. Por ello, la política impulsada postula un comercio internacional no discriminatorio, transparente, flexible, basado en la eficiencia y en el desarrollo de las ventajas comparativas. ${ }^{12}$

El polo de gravitación al instalarse el gobierno de Patricio Aylwin, en marzo de 1990, estuvo centrado en los aspectos políticos, es decir "reinsertar al país en el sistema internacional". Esta meta fue completada rápida y eficientemente. Desde 1991, es la política económica internacional la que adquirió una mayor gravitación. La estrategia de desarrollo nacional está basada en la creciente internacionalización de la economía: el 38\% del producto geográfico está vinculado al sector exportador y la meta del gobierno de transición es que esta cifra alcance el $40 \%$ en 1994.

Las relaciones económicas con Estados Unidos poseen una alta prioridad. En 1989, Chile exportaba a los Estados Unidos un 17,8\% y cerca de un $22,0 \%$ de sus importaciones las efectuaba desde ese mercado. ${ }^{13}$ Estados Unidos constituía, a inicios del proceso de transición, el primer socio comercial individual del país. En 1991, la situación ha cambiado y Japón se ha transformado en el principal socio comercial del país. Estados Unidos es el destino de un $17.6 \%$ de las exportaciones y allí tienen origen un $20.2 \%$ de las importaciones.

La Iniciativa de las Américas fue recibida con "especial beneplácito" por el gobierno chileno, lo cual se tradujo en un curso de acción rápido: aceptar la propuesta de establecer una zona de libre

\footnotetext{
${ }^{12}$ Carlos Portales, "La política exterior chilena en el nuevo contexto político y económico internacional", Cono Sur, Vol. XI, N2 1, FLACSO-Chile, enero-febrero 1992.

${ }^{13}$ Banco Central de Chile, Indicadores de Comercio Exterior, Santiago de Chile, abril 1992.
} 
comercio y comenzar en forma inmediata la negociación. Además, inicialmente se evaluó que un rápido avance podría facilitar la resolución de otros temas de la agenda bilateral, en especial los referidos a las restricciones no arancelarias y al escalonamiento arancelario que tienen los productos nacionales en el mercado norteamericano.

La rápida aceptación de la propuesta norteamericana significó redefinir las prioridades de la diplomacia económica chilena. En los meses iniciales de gobierno la prioridad había estado puesta en América Latina, ya que se trataba de recuperar el espacio político en la región. La reinserción se alcanzó y se consolidó con el ingreso al Grupo de Río. En lo económico, se trataba de revertir el déficit regional y de generar oportunidades de negocios en los países del área.

En lo bilateral, priorizar la negociación con Estados Unidos significó definir un curso de acción propio de carácter unilateral. La otra opción era establecer un camino conjunto con los países latinoamericanos. Sin embargo, se definió que era necesario tomar la iniciativa y no esperar. Cabe destacar que las autoridades norteamericanas consideran que el establecimiento de la zona de libre comercio hemisférica es una meta de largo plazo, dado que este proceso demorará una década, si no más. ${ }^{19}$ Habiéndose percibido tempranamente esta situación, concertarse implicaba perder oportunidades ya que las condiciones para ello significaban que había que esperar que el ajuste económico fuese realizado en los diversos países. Al existir una gran variedad de políticas y de ritmos de avance en la apertura -y de oposición a ella-, la concertación frente a la Iniciativa para las Américas se transformaba en inacción y en la pérdida de una oportunidad para que el país avanzara hacia un acuerdo con la mayor economía del hemisferio. No obstante, las gestiones para llegar a entendimiento con los países de la región deberían continuar. Luego del anuncio de la Iniciativa de la Casa Blanca, desarrollar un programa de acción que llegase a un Acuerdo de Libre Comercio con Estados Unidos ocupó un lugar central de la agenda y una mayor energía burocrática.

Dentro de las actividades de la primera visita del Presidente Aylwin a Washington, y como consecuencia de las acciones y decisio-

\footnotetext{
${ }^{14}$ Embajada de Estados Unidos en Chile. "Documento informativo del Departamento de Comercio sobre la Iniciativa para las Américas", Documento de Consulta, 26 de septiembre de 1991.
} 
nes desarrolladas con Estados Unidos, el Ministro de Hacienda, Alejandro Foxley, y la Representante Comercial de los Estados Unidos, Carla Hills, firmaron el $1^{\circ}$ de octubre de 1990 un Acuerdo Marco para los temas económicos. Por medio de este acuerdo se creó un Consejo Bilateral de Comercio e Inversión, que tiene como misión abordar los problemas concretos de las relaciones económicas bilaterales y avanzar hacia un Tratado de Libre Comercio. También se definió una Agenda de Acción Inmediata, dentro de la cual uno de los temas centrales es establecer la cooperación en las negociaciones multilaterales del GATT. Fue en el contexto de la visita del Presidente estadounidense en el mes de diciembre de 1990, que las relaciones quedaron normalizadas en lo que a las sanciones heredadas se refiere, y se avanzó en la posibilidad de suscribir un acuerdo comercial bilateral.

En marzo de 1991, cuanđo el Presidente Bush solicitó al Congreso la prórroga del procedimiento de "vía rápida", mencionó que dentro de él podrían caber las negociaciones con Chile. Esto fue calificado como un gran respaido a la política desarrollada por el Presidente Aylwin.

Como parte de los beneficios de la política y las acciones desarrolladas en la perspectiva de un Acuerdo de Libre Comercio con Estados Unidos, Chile se benefició en un $40 \%$ de la reducción de su. deuda oficial, correspondiente al PL 480. En el acto de celebración del primer año de la Iniciativa, el Ministro Foxley y el Secretario de Estado Brady, con la presencia del Presidente Bush, suscribieron un acuerdo para reducire la deuda oficial del país en 16 millones de dólares.

En Santiago y Washington, se han reunido alternativamente en cuatro oportunidades los equipos técnicos de ambos países. Se creó un lobby, se han iniciado los estudios sobre los posibles efectos del Acuerdo de Libre Comercio y diversas misiones estadounidenses han visitado el país. Luego de la reunión ministerial entre Alejandro Foxley y Carla Hills en noviembre de 1991, queđó establecido el mutuo interés en avanzar en el proceso negociador. Sin embargo, esta negociación será finiquitada una vez concluida la negociación norteamericana con México y Canadá. Las autoridades chilenas

\footnotetext{
15 Ver declaraciones del Embajador Patricio Silva, 3 de marzo de 1991. En: "De la Reinserción a los Acuerdos. La Política Exterior Chilena en 1991", FLACSO-Chile, Santiago, 1992.
} 
confían que el acuerdo quedará incluido en los tiempos establecidos por el Congreso norteamericano para aprobar o rechazar las propuestas gubernamentales por una "vía rápida". La suscripción del acuerdo quedó por lo tanto condicionada al timing del NAFTA.

Con la visita de Estado del Presidente Aylwin del mes de mayo de 1992, concluyó una etapa en las relaciones con Estados Unidos y la cooperación y la búsqueda de acuerdos pasó a ser el patrón dominante. Se formalizó al máximo nivel gubernamental la voluntad política de establecer un Acuerdo de Libre Comercio.

En la declaración emitida por la Casa Blanca; el 13 de mayo de 1992, se manifestó la voluntad política y burocrática de firmar un acuerdo con Chile dentro de la Iniciativa de las Américas y en un "tiempo apropiado". Este fue ubicado al momento de "concluir" la negociación del NAFTA. Sin embargo, no está definido con claridad lo que significa "concluida" la negociación. Se entiende que el tiempo apropiado está referido a que la apertura de negociaciones formales con Chile no debería poner en riesgo ese acuerdo fundamental.

Hasta la fecha se han realizado algunos estudios que buscan evaluar el impacto potencial de un posible Acuerdo de Libre Comercio, siendo diversos sus resultados. El Banco Mundial estima que para el caso de Chile el incremento comercial podría alcanzar un 6\%. De este modo, desde el punto de vista estrictamente comercial Chile, tendría una expansión marginal con un acuerdo de este tipo con Estados Unidos.

En particular, es en la inversión donde se destaca la importancia de un Acuerdo de Libre Comercio. ${ }^{16} \mathrm{La}$ suscripción posibilitaría un incremento sustancial de la inversión extranjera en el país. Ello significa que la economía chilena es definida como confiable, estable, creibley transparente. El debate hoy día existente sobre la posibilidad de suscribir un Acuerdo de Libre Comercio con Estados Unidos, nos acerca a esta definición conceptual de la economía nacional.

Establecer un régimen específico para la solución de controversias es uno de los principales objetivos al suscribir el Acuerdo. La conformación de un régimen específico de carácter simétrico en una relación asimétrica, es un gran logro para el actor más débil en la relación.

\footnotetext{
${ }^{16}$ Andrea Butelmann y Alicia Frohmann, "Hacia un Acuerdo de Libre Comercio entre Chile y Estados Unidos", Relaciones Económicas Internacionales Chile/Estados Unidos, No3, FLACSO/ CIEPLAN, Santiago de Chile, 1992.
} 
El impacto del Acuerdo de Libre Comercio es crucial en lo político, significativo en lo económico e importante en lo comercial. Esta escala de magnitud y el horizonte de impacto temporal en el cual se ubica, es lo que debe estar presente en el momento de la toma de decisiones. Esta corresponderá, dado el timing de la negociación, a los próximos gobiernos en Estados Unidos y en Chile.

En general, la política seguida ha tenido un fuerte sello gubernamental a la que se ha ido incorporando crecientemente un importante sector del empresariado. Pero han estado fuera del debate importantes actores, como lo son el Parlamento y los trabajadores. La construcción de una política de Estado se expresará en la incorporación de estos y otros actores, en un debate nacional sobre el tema que aún está pendiente.

\section{El Mercado Común del Sur (MERCOSUR).}

El MERCosur es un mercado de alrededor de 200 millones de personas y con un producto superior a los 500 mil millones de dólares. Representa, hoy día, el $65 \%$ de la población sudamericana, más del $76 \%$ de su producto bruto, más del $60 \%$ del industrial y más del $53 \%$ de su comercio exterior. En 1990, el $70 \%$ del stock de la inversión acumulada en América Latina de origen europeo y japonés, estaba concentrada en los cuatro países. El MERcosur es sin duda un gran "megamercado", con una inserción múltiple en la economía global. ${ }^{17}$ Su debilidad está en la fragilidad de los procesos de ajuste económico y la inestabilidad política del gobierno de Brasil en la actual coyuntura, como producto del proceso de recambio presidencial luego de la positiva y ejemplarizante acción fiscalizadora del Congreso. Ello dificulta las opciones para aprovechar en forma dinámica las potencialidades de ese "megamercado". Uno de los cambios principales que ha producido el MERCOSUR ha ocurrido en el sector privado empresarial. De una actitud inicial de clara desconfianza y descrédito, está evolucionando hacia una posición de seguimiento interesado y en algunos sectores de involucramiento directo. ${ }^{18}$

\footnotetext{
${ }^{17}$ Félix Peña, "La cumbre de las leñas y el futuro del MERcosur, Ministerio de Relaciones Exteriores, Buenos Aires, Argentina, julio 1992.

${ }^{18}$ Rubens A. Barbosa, "MERcosul: Baianço do Primeiro Ano", Boletim de Integraçáo Latino-Americana, Ministério das Relaçóes Exteriores, №4, Brasilia, Brasil, 1992.
} 
Los países del Cono Sur tienen una inserción múltiple en la economía global y sus exportaciones no se concentran en un solo polo de destino, lo que es claro para Chile y los países del MERCosur. El comercio interregional es aún débil y puede crecer significativamente. Para la Argentina, las exportaciones al MERCosur representan sólo el 13\% de sus exportaciones totales y para el Brasil, apenas el $6 \%$. Los Estados Unidos representan el $12 \%$ de las exportaciones argentinas y el $20 \%$ de las de Brasil. Los cuatro países del Mercosur destinan el 33\% de lo que exportan al conjunto de la Comunidad Europea. La ejecución de los acuerdos que fomentan la desgravación y la integración han tenido importantes éxitos, y es así como el 30 de junio de 1992 el margen de preferencia para el MERCOSUR aumentó del 54 al $61 \%$. En diciembre de 1994 se habrá completado el proceso de apertura en este importante mercado del Cono Sur. ${ }^{19}$

Con este pacto subregional, Estados Unidos firmó un acuerdo marco conocido como "cuatro por uno", reconociendo el proceso de integración del MERcosur. De este modo, se eliminaron las hipótesis iniciales que plantearon que la Iniciativa para las Américas era una forma de debilitar y destruir la integración regional. En la Casa Blanca, el 19 de junio de 1991, fue suscrito un acuerdo similar a los otros bilaterales. Por medio de éstos se crearon los instrumentos del caso, como es el Consejo Consultivo sobre Comercio e Inversión y se estableció una agenda inmediata. En el contexto de este acuerdo se han celebrado dos reuniones técnicas de trabajo.

Uno de los objetivos de los países del Mercosur fue incorporar otro temas en la agenda de la Iniciativa para las Américas o ampliar el rango de acción de alguno de ellos. La transferencia de tecnologías y la deuda son dos de las materias fundamentales. Asegurar capacidades futuras significa incorporar los avances y los saltos generacionales de desarrollo tecnológico. Resolver el problema de la deuda externa es clave para Brasil y Argentina, dos de los países más endeudados de la región.

Estos dos puntos que reflejan los intereses de los países del Cono Sur, fueron señalados como aspectos insalvables por Estados Unidos y no podían ser incorporados como parte del acuerdo marco. Las razones esgrimidas fueron que el primero estaba referido a restricciones de seguridad nacional y el segundo porque existía un

\footnotetext{
${ }^{19}$ Félix Peña, op. cit.
} 
mecanismo de solución, el Plan Brady. No es claro que ambos puedan ser parte de la agenda de discusión entre el bloque regional y la potencia hemisférica.

\section{Argentina. ${ }^{20}$}

La política exterior argentina cambió radicalmente. Hoy, bajo la presidencia de Carlos Menem, el eje de la vinculación con el exterior es el alineamiento con Washington, lo que se expresa en el conjunto de acciones desarrolladas. Estos cambios se orientarían por la "desideologización" de las relaciones internacionales. Una de las mayores expresiones de esta política fue la participación de Argentina-único país latinoamericano-en la Guerra del Golfo.

En forma paralela, Argentina ha desarrollado un importante programa de ajuste económico, apertura de mercado, liberalización del comercio exterior, reducción y eliminación de subsidios, y una amplia política de privatización. Se garantiza trato nacional a la inversión externa y su economía se ha dolarizado. Todo ello ha redundado en una drástica reducción de la inflación, una recuperación de la inversión y la extensión de un clima de confianza para los negocios.

En Argentina, se percibe que estos cambios serán exitosos ya que son coincidentes con los cambios globales y, en especial, con el nuevo espacio que estaría encontrando la región latinoamericana en la política de Estados Unidos. La tendencia al regionalismo mundial encuentra al país en buena posición para consolidar su acercamiento a los Estados Unidos.

El país recibió con gran entusiasmo la propuesta de la Iniciativa para las Américas. El Presidente Menem la calificó como "un paso trascendental en el aumento de la prioridad de América Latina en la política exterior de Estados Unidos". Sin embargo, sus resultados no serán inmediatos.

En el sector empresarial se indica que las exportaciones están poco concentradas en el mercado norteamericano y que los aumentos potenciales en este momento tienen dos tipos de dificultades: una,

\footnotetext{
${ }^{20}$ Rut Diamint, "La Iniciativa para las Américasy la Argentina", en: Francisco Rojas Aravena (ed.) La Respuesta Latinoamericana frente a la Iniciativa para Ias Américas, FLACSO-Chile, (por publicarse).
} 
derivada de las restricciones de ingreso al mercado norteamericano y otra, la de mayor significación, basada en la dificultad de aumentar la producción argentina orientada hacia éste.

El mercado norteamericano es el destino del $13 \%$ de las exportaciones. A la Comunidad Europea se dirige el $30 \%$ de ellas y un $25 \%$ se coloca en los países de la región.

La Iniciativa para las Américas es percibida como una invitación a actuar, pero hay que tener en cuenta que los resultados se verán recién a mediano plazo. Ello significa que los argentinos deberán adecuarse a una nueva situación en la cual es posible preveer y programar negocios a mediano y largo plazo.

\section{Brasil. $^{21}$}

Para Brasil lo fundamental es que los esfuerzos integracionistas no son incompatibles con un mayor acercamiento hemisférico. La integración es hoy parte de las demandas del ajuste y de la modernización de la economía.

En los países en que no se ha realizado el ajuste o en donde éste ha sido parcial -como es el caso de Brasil-, no se puede producir la apertura y generar las condiciones para una nueva inserción productiva, sin que los partidos de gobierno deban pagar un costo político doméstico de significación. Ello genera resistencias; se acepta la retórica del cambio pero no se acepta o se busca bloquear los efectos y cambios institucionales y políticos que pueden afectar lo estructural.

En los últimos años, Brasil ha buscado ampliar sus oportunidades de inserción. Ello significa actuar en el ámbito multilateral, producir una apertura a las corrientes internacionales de comercio e inversión y buscar un balance en las cuentas internacionales. El país tiene ampliamente diversificado el destino de sus exportaciones: en 1989 exportaba un $30 \%$ a la Comunidad Europea, un $25 \%$ a Estados Unidos, un $10 \%$ a América Latina y un 7\% a Japón. La percepción brasileña sobre la Iniciativa de las Américas debe ser visualizada teniendo presente esta formalización.

\footnotetext{
${ }^{21}$ Alcidez Costa Vaz, "La Iniciativa para las Américas desde la perspectiva brasilera", en: Francisco Rojas Aravena (ed.), La Respuesta Latinoamericana frente a la Iniciativa para las Américas, FlACSO-Chile, (por publicarse).
} 
La Iniciativa no representa por sí sola -más allá de los aspectos positivos en ella contenidos- una alternativa de inserción económica para Brasil. Tampoco resuelve la relación con los Estados Unidos, debido a que la agenda bilateral es mucho más compleja aún si consideran sólo los aspectos económicos. Además, de los tres aspectos involucrados en la Inicitiva, la agenda bilateral brasileña-norteamericana comprende temas tales como transferencia de tecnologías, medio ambiente, propiedad de intelectual y protección a las exportaciones.

La oficina del representante comercial de los Estados Unidos (USTR) ha excluido muchos de estos temas de la discusión, en particular lo relativo a la tecnología.

Brasil recibió con entusiasmo la Inicitiva para las Américas. Sin embargo, el fervor inicial se fue enfriando al percibirse sus límites en relación a los intereses nacionales brasileños. La posición del gobierno brasileño es cada vez más pragmática y la posición del empresariado es cautelosa. La percepción que engloba esto indica que Estados Unidos busca resolver "sus" problemas, más que los problemas comerciales latinoamericanos y, además, intenta captar el apoyo regional para su política en el GATT.

Inicialmente, se percibió que la Iniciativa para las Américas permitiría establecer un nuevo canal de entendimiento con los Estados Unidos capaz de resolver problemas que fueran más allá del ámbito comercial. Sin embargo, la exclusión de temas claves para Brasil como la transferencia tecnológica, la deuda externa o la compatibilidad legal con la ALADI, hicieron perder fuerza a las percepciones más optimistas. Además, se debe considerar que el país quedó en una situación de status quo como producto de la acusación constitucional - por corrupción- al Presidente Collor. Ello eliminó otras prioridades de la agenda y el país se concentró en la resolución de sus problemas domésticos.

Se ha avanzado en estudios sobre el impacto que puede tener la Iniciativa que señalan que una mayor presencia norteamericana significará concurrencia en el mercado local y competencia en el mercado regional. Esto en un contexto en el cual el comercio bilateral brasileño-norteamericano muestra una tendencia al descenso, afectando ya a un $10 \%$ de las exportaciones de Brasil, en especial, de productos manufacturados.

En relación al tema de la deuda -si bien es clave para Brasil-el hecho de que ésta sea negociada caso a caso, produce incertidumbre 
sobre las condiciones que se aplicará finalmente a cada país. Brasil no acepta que la transformación de su deuda en fondos ambientales pueda servir de plataforma para una reducción de su soberanía sobre el Amazona.

\section{Conclusiones.}

América Latina y el Cono Sur enfrentan la nueva etapa de post Guerra Fría en un contexto de regímenes políticos ampliados que buscan avanzar y consolidar los logros democráticos. En lo económico, los países de la región han realizado un importante proceso de apertura que busca establecer una nueva forma de inserción internacional. Sin embargo, los logros en ambas esferas no se han consolidado. La gobernabilidad en la región está referida a la capacidad de las elites políticas, empresariales, sindicales y militares, de articular la participación democrática con la disciplina macroeconómica a la vez que se toman medidas para solventar los problemas de los sectores de más escasos recursos. Esta es una ecuación que no posee una sola respuesta y en ella inciden de manera cada vez más determinante los fenómenos globales. De allí el interés con que los países de la región recibieron la Iniciativa para las Américas.

La Iniciativa representa un gran cambio: pasar desde una opción constreñida por el conflicto ideológico a la oportunidad de cooperación comercial, como base para una nueva forma de relación hemisférica. En la nueva etapa donde las relaciones económicas tienden a tener primacía sobre las de seguridad, al menos a nivel global, es una señal positiva para la creación de oportunidades de cooperación y asociación.

Con el fin de la Guerra Fría, es posible trabajar para establecer un nuevo patrón de relación entre el poder hegemónico del hemisferio y los países latinoamericanos, en general, y los del Cono Sur, en particular. El tipo de relación que es factible establecer puede fundarse en la asociación, pero ello no es automático. Es necesario incidir en la agenda, establecer principios y concertar políticas. Para avanzar se debe afianzar la concertación política del Cono Sur, ya que sin ella se dificulta la aplicación de políticas que tengan incidencia efectiva y que se transformen en un incentivo de cooperación y asociación para los Estados Unidos. La experiencia de la Iniciativa 
para las Américas y la respuesta regional, nos señalan las grandes dificultades para conciliar y organizar los intereses nacionales de los países latinoamericanos. La coincidencia temporal de gobiernos democráticos y el desarrollo de políticas de apertura no son suficientes.

El análisis de casos nos muestra que la respuesta del Cono Sur -y más claramente cuando se compara con el Caribe y Centroamérica- fue prematura, diversa, dispersa, desinformada y desarticulada. Fue prematura, porque no hubo un análisis en profundidad de las posibilidades efectivas de implementar la Iniciativa, y de si ésta buscaba tener impacto internacional o más bien era de carácter doméstico, vinculada al punto central: el NAFTA. Fue diversa, ya que no hubo concertación para responder como subregión. El tipo de acción generada se basó en las capacidades individuales y la dispersión restó fuerza a una opción de efectiva negociación o para incluir temas en la agenda. La información sobre el sistema político norteamericano y sus procesos decisorios, que era clave en la evaluación dei proceso, parece no haber tenido un peso significativo en la respuesta regional. Cada uno de estos puntos se fue reforzando mutuamente y ello significó que la región latinoamericana actuara en forma desarticulada. La respuesta regional y del Cono Sur se fragmentó y con ello perdió capacidad de incidencia. A la vez, la fragmentación tendió a incrementar la competencia más que la búsqueda de respuestas conjuntas.

Este es uno de los ámbitos donde la concertación regional ha mostrado sus falencias y donde la diversidad de intereses se evidencia con claridad. La competencia por las inversiones, por espacios privilegiados en el mercado norteamericano y por ser "países modelo" sujetos de recibir recompensas, redunda en la falta de concertación multilateral. Con ello se refuerza el bilateralismo tradicional norteamericanoy se debilitan las capacidades para generar mejores espacios para la negociación. De hecho el desarrollo de varias negociaciones bilaterales refuerza las capacidades del centro en un modelo radial (hub and spoke).

La compatibilidad de intereses latinoamericanos no quedó en evidencia y no se crearon espacios para la generación de alternativas. La permanencia de la recesión y ciertas tendencias proteccionistas en Estados Unidos, restaron peso político a la Iniciativa para las Américas y fue necesario concentrarse en el NAFTA. Ello reforzó la diferenciación latinoamericana y los diversos países buscaron ser el 
"segundo en la línea" de ingreso, más que intentar coordinar políticas. Concluido el NAFTA, no es seguro que quede espacio para continuar con la Iniciativa. Si se miran los cambios estructurales, se debe constatar que la apertura política y económica de la región le permiten una nueva forma de relación -aun bilateral-con Estados Unidos y los principales centros desarrollados. Queda pendiente la tarea de buscar formas de concertación regional para alcanzar metas en el sistema internacional.

\section{REFERENCIAS BIBLIOGRAFICAS}

Agosín, Manuel R., "Las Experiencias de Liberalización Comercial en América Latina: Lecciones y Perspectivas", Pensamiento Iberoamericano, Vol. Especial, № 21, 1992.

Banco Central de Chile, Indicadores de Comercio Exterior, Santiago de Chile, abril de 1992.

Barbosa, Rubens A., "MERCosul: Balanço do Primer Ano", Boletim de Integraçáo Latinoamericana, Ne 4, Ministerio de Relaciones Exteriores, Brasilia, Brasil, 1992.

Butelmann, Andrea y Campero, Pilar, "Escalonamiento arancelario para las exportaciones chilenas a los EE.UU.", Notas Técnicas, No147, CIEPLAN, Santiago de Chile, mayo 1992.

Butelmann, Andrea y Frohmann, Alicia, "Hacia un Acuerdo de Libre Comercio", CIEPLAN/FLACSO, №3, Santiago de Chile, febrero 1992.

Costa-Vaz, Alcides, "La Iniciativa para las Américas desde la perspectiva brasileña", en: Francisco Rojas Aravena (ed.), América Latina y la Iniciativa para las Américas, FLACSO-Chile, (por publicarse).

Diamint, Rut, "La Iniciativa para las Américas y la Argentina", en: Francisco Rojas Aravena (ed.), América Latina y la Iniciativa para las Américas, FLACso-Chile, (por publicarse).

"Discurso pronunciado por el Presidente Bush el 27 de junio sobre Nuevas Relaciones con el Hemisferio", Materiales de Consulta, Embajada de Estados Unidos, 28 de junio de 1990.

"Enterprise for the Americas: Vision and Reality", en: North-South ISSUES (Trade), University of Miami, Florida, July 1992.

Erzan, Refik \& Yeats, Alexander, "Free Trade Agreements with the United States. What's In It for Latin America?", Working Papers, International Economics Department, The World Bank, January 1992. 
FLACSO, "La reinserción internacional de Chile", Serie Política Exterior y Relaciones Internacionales, Area de Relaciones Internacionales, FLACSO, 1991.

FLACSO, "Iniciativa para las Américas. Actores, Hechos, Audiencias'en el Congreso de Estados Unidos", Proyecto de Investigación y Seguimiento de la Iniciativa para las Américas, Flacso, Santiago de Chile, enero 1992.

Flacso, "De la Reinserción a los Acuerdos", Serie Política Exterior y Relaciones Internacionales, Area de Relaciones Internacionales, FLACSO, 1992.

Fuentes K., Juan Alberto, "Compatibilidad entre la integración subregional y la hemisférica", Revista de la CEPAL, №45, Santiago de Chile, diciembre 1991, pp. 107-128.

Maira, Luis, "Un examen de la Iniciativa para las Américas en el contexto del proceso político norteamericano", Proyecto Iniciativa para las Américas, Area de Relaciones Internacionales, ILET, 1992.

"North American Free Trade Agreement", August, 1992.

Peña, Félix, "La cumbre de las leñas y el futuro del MERCosur", mimeo, Ministerio de Relaciones Exteriores, Buenos Aires, Argentina, 1992.

Portales Cifuentes, Carlos, "La política exterior chilena en el nuevo contexto político y económico internacional", Cono Sur, Vol. XI, № 1, FLACSO-Chile, Santiago de Chile, enero-febrero 1992.

Porteer, Roger B., "The Enterprise for the Americas Initiative a New Approach to Economic Growth", Joumal of Interamerican Studies and World Affairs, Vol. 32, № 4, Miami, Estados Unidos, Winter 1990.

Rojas Aravena, Francisco, "De la reinserción a los acuerdos: la política exterior chilena en 1991", Cono Sur, Vol. XI, No 1, FLACso-Chile, Santiago de Chile, enero-febrero 1992.

Rojas Aravena, Francisco, "Chile y la Iniciativa para las Américas", FLACSOChile, Santiago de Chile, 1992, (original no publicado).

SELA, "Análisis y comentarios sobre el proyecto de Acta Final de la Ronda de Uruguay", Secretaría Permanente, SELA, 14 de abril de 1992.

Tulchin, Joseph S., "Enterprise for the Americas Initiative. Iniciativa para las Américas", Proyecto de Investigacion y Seguimiento, The Latin American Program, The Woodrow Wilson Center, 1992. 